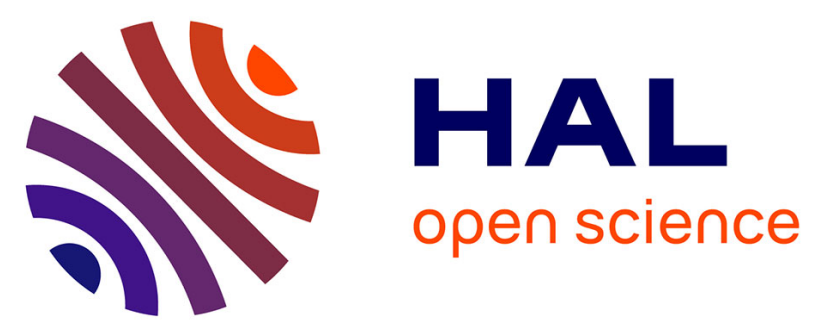

\title{
Probing plasmonic hot spots on single gold nanowires using combined near-field techniques
}

\author{
Patrick Hsia, Ludovic Douillard, Fabrice Charra, Sylvie Marguet, Serguei \\ Kochtcheev, Renaud Bachelot, Céline Fiorini-Debuisschert
}

\section{- To cite this version:}

Patrick Hsia, Ludovic Douillard, Fabrice Charra, Sylvie Marguet, Serguei Kochtcheev, et al.. Probing plasmonic hot spots on single gold nanowires using combined near-field techniques. Proceedings of SPIE, the International Society for Optical Engineering, 2015, Proc. SPIE 9547, Plasmonics: Metallic Nanostructures and Their Optical Properties XIII, 95470F (28 August 2015), 9547, $10.1117 / 12.2188025$. cea-01484784

HAL Id: cea-01484784

https://hal-cea.archives-ouvertes.fr/cea-01484784

Submitted on 15 Mar 2017

HAL is a multi-disciplinary open access archive for the deposit and dissemination of scientific research documents, whether they are published or not. The documents may come from teaching and research institutions in France or abroad, or from public or private research centers.
L'archive ouverte pluridisciplinaire HAL, est destinée au dépôt et à la diffusion de documents scientifiques de niveau recherche, publiés ou non, émanant des établissements d'enseignement et de recherche français ou étrangers, des laboratoires publics ou privés. 


\title{
Probing plasmonic hot spots on single gold nanowires using combined near-field techniques
}

\author{
Patrick Hsia ${ }^{a}$, Ludovic Douillard ${ }^{\mathrm{a}}$, Fabrice Charra ${ }^{\mathrm{a}}$, Sylvie Marguet ${ }^{\mathrm{b}}$, Sergei Kostcheev ${ }^{\mathrm{c}}$, Renaud \\ J.B. Bachelot ${ }^{\mathrm{c}}$, Céline Fiorini-Debuisschert ${ }^{\mathrm{a}}$, \\ a Nanophotonics Laboratory, CEA Saclay IRAMIS/SPEC - CNRS UMR 368091191 Gif-sur-Yvette \\ Cedex, FRANCE; 'Francis Perrin lab. - CEA Saclay IRAMIS/NIMBE - CNRS URA 2453, 91191 \\ Gif-sur-Yvette, France; ${ }^{\mathrm{C}}$ Institut Charles Delaunay, LNIO, 10010 Troyes, FRANCE
}

\begin{abstract}
The plasmonic properties of individual gold nanowires (NW) have been investigated using both two-photon luminescence (2PL) coupled to atomic force microscopy (AFM) and photoemission electron microscopy (PEEM) associated to low-energy electron microscopy (LEEM) measurements. Using these complementary near-field characterization techniques, comparative studies between wires made either by colloidal chemistry (CC) or by e-beam lithography (EBL) have been undertaken towards a better understanding of the role of the wires crystallinity regarding its optical properties. Considering comparable excitation conditions, we show that wires made by colloidal synthesis exhibits quite similar field enhancement effects ("hot spots") as EBL NW, however their 2PL emission spectrum clearly reveals their crystalline properties.
\end{abstract}

Keywords: Two-photon luminescence, AFM, gold nanowire, plasmonic hot spots, crystallinity, PEEM/LEEM

\section{INTRODUCTION}

Plasmonics is currently a hot topic in the field of nano-optics. Surface plasmon resonances (SPR) refer to the collective oscillations of conduction electrons driven by an external electromagnetic field at the interface between a metallic and a dielectric medium. These resonances are highly dependent on the excitation wavelength and lead to a spatially confined field enhancement (FE). These localized zones - called hot spots - find applications in a large variety of fields such as photovoltaics ${ }^{1}$, nanosensors ${ }^{2}$ or nanolasers ${ }^{3}$. Nanostructures made by noble metals (such as silver or gold) are known to sustain such oscillations and with simple geometries, the SPR can rather easily be determined ${ }^{4}$. Among them, nanowires (NW) exhibit very interesting properties due to their anisotropic shape leading to two resonances: a transversal resonance in the visible and a longitudinal resonance in the near infrared, this longitudinal resonance being directly tunable through the NW aspect ratio.

Gold NW can more particularly be the place of different processes leading to field enhancement:

- a lightning rod effect at the extremities of the NW, provided that the extremities of the NW are shaped like sharp tips. This geometric singularity effect depends on the polarization of the incident laser. To maximize this non-resonant effect, the direction of the incident polarization has to be aligned with the tip axis ${ }^{5}$.

- a localized surface plasmon resonance also leading to hot spots at the NW extremities. Such resonance highly depends on the excitation wavelength,

- for longer wires, Fabry-Perot like resonances due to surface plasmon polaritons propagating along the wire (model of half-wave dipole antenna). These resonances are ruled by the following equation $(1)^{6,7}$.

$$
2 L=m \lambda_{\text {eff }}=m \frac{\lambda_{0}}{n_{\text {eff }}},
$$

with $L$ : the length of the wire, $m$ : the order of resonance considered ( $m$ is an integer), $\lambda_{\text {eff }}$ is the effective wavelength which depends on the material used, $\lambda_{0}$ : the excitation wavelength and $n_{\text {eff: }}$ the effective index of the medium which also depends on the material and the surrounding ${ }^{6}$. 
Together with analyzing the different contributions to FE effects in NW, the objective of the work presented here was to study the effect of the NW crystallinity on their plasmonic properties.

For this purpose, two kinds of NW were considered: NW synthetized by colloidal chemistry (CC) with well-defined crystalline faces and NW made by electron beam lithography (EBL) exhibiting surface roughness inherent to the lithography process, as revealed by scanning electron microscopy (SEM - figure 1).
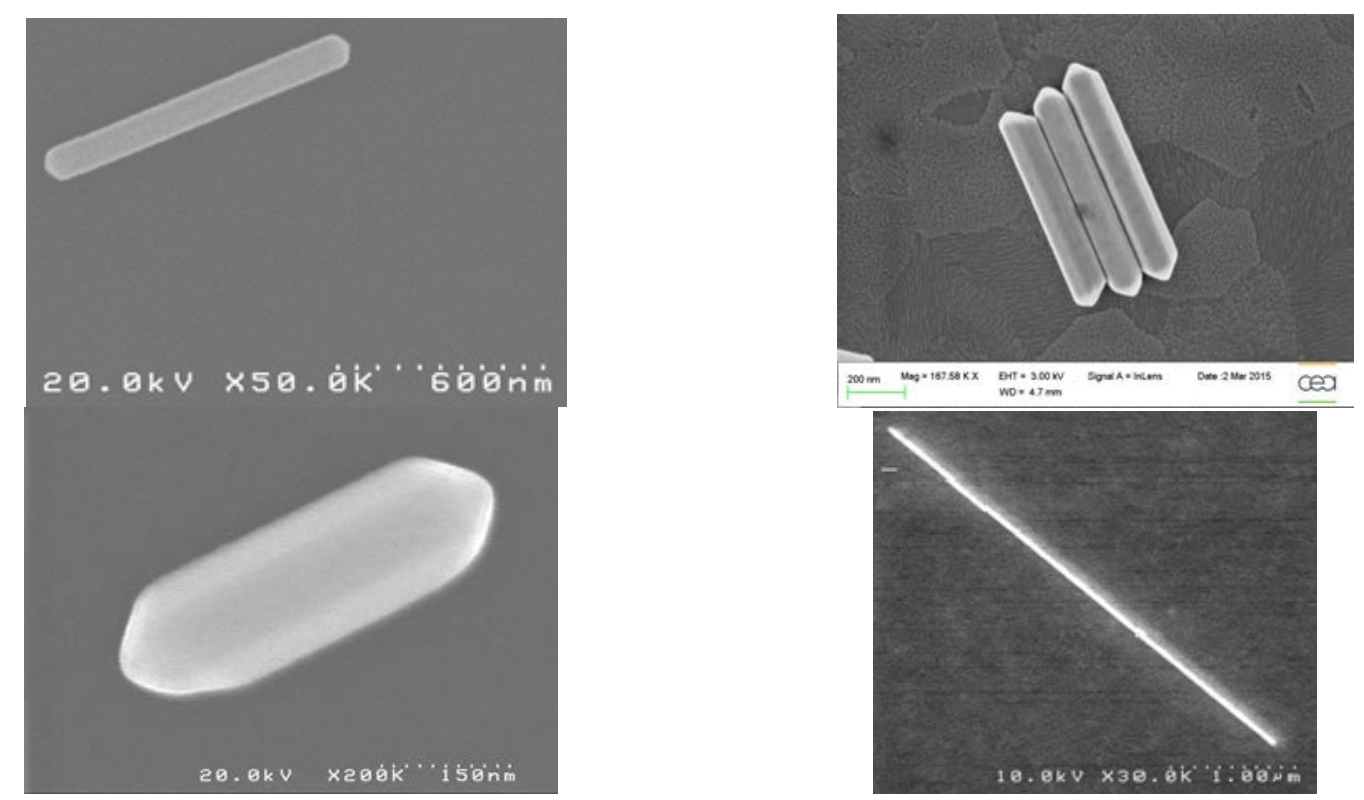

Figure 1: (Up Left) SEM image of a 945 nm x 105 nm gold NW synthetized by CC. (Up Right) Different NW showing different crystalline faces (bi-twinned, penta-twinned). (Down Left) SEM image of another NW synthetized by CC with sharper ends (483 nm x $156 \mathrm{~nm}$ ). (Down Right) SEM image of a $4 \mu \mathrm{m}$ x $35 \mathrm{~nm}$ gold NW made by EBL. The average surface roughness is about $10 \mathrm{~nm}$.

$4 \mu \mathrm{m}$ long and $200 \mathrm{~nm}$ width NW were directly fabricated onto ITO glass cover slides using standard EBL techniques. Although the reproducibility of the structures size is quite good, the fabrication process was however observed to lead to quite important variations in the NW roughness and average thickness. In the case of CC NW, a specific protocol was developed in order to get stable NRs immobilization and to avoid any aggregation: ITO coated glass substrates were first carefully cleaned and further treated using UV ozone irradiation. This treatment is indeed known to lead to the formation of negatively charged hydroxyl groups at the ITO surface enabling consequently electrostatic coupling of the gold NW given the net positive charge coming from the presence of the cationic surfactant resulting from their fabrication (cetyltrimethylammonium (CTAB) $)^{8}$ A droplet of the NW solution of a given concentration was then deposited directly onto each freshly treated slides. The droplet was left to dry, then washed thoroughly with water and ethanol, to eliminate either uncoupled nanoparticles or CTAB in excess. Due their fabrication process, CC NW were observed to be rather polydisperse with lengths typically varying between a few hundred of $\mathrm{nm}$ to $1 \mu \mathrm{m}$ and widths ranging from 50 to 200 $\mathrm{nm}$. The NW can be localized by using grids made by EBL, each unit cell is a $50 \mu \mathrm{m} \times 50 \mu \mathrm{m}$ square and can uniquely be identified by the association of a letter corresponding to a column and a digit corresponding to a row. In this way, preliminary SEM characterizations can be undertaken before the optical characterization. 


\section{EXPERIMENTAL SETUPS FOR PROBING PLASMONIC HOT SPOTS}

Plasmons are known to decay either radiatively through photon emission (luminescence) or non-radiatively, through e.g. photoelectron emission. As further detailed below, these two relaxation pathways have been taken into profit to map the hot spots at the surface of the NW considered in this study.

\section{2-1 Simultaneous 2-photon luminescence (2PL) and topography characterization}

Although the origin of luminescence in gold nanostructures is still matter of debate ${ }^{9}$, 2PL was shown to be directly linked to the local density of electromagnetic states $(\mathrm{LDOS})^{10}: 2 \mathrm{PL}$ thus constitutes a relevant tool to probe the local hot spots on nanostructures.

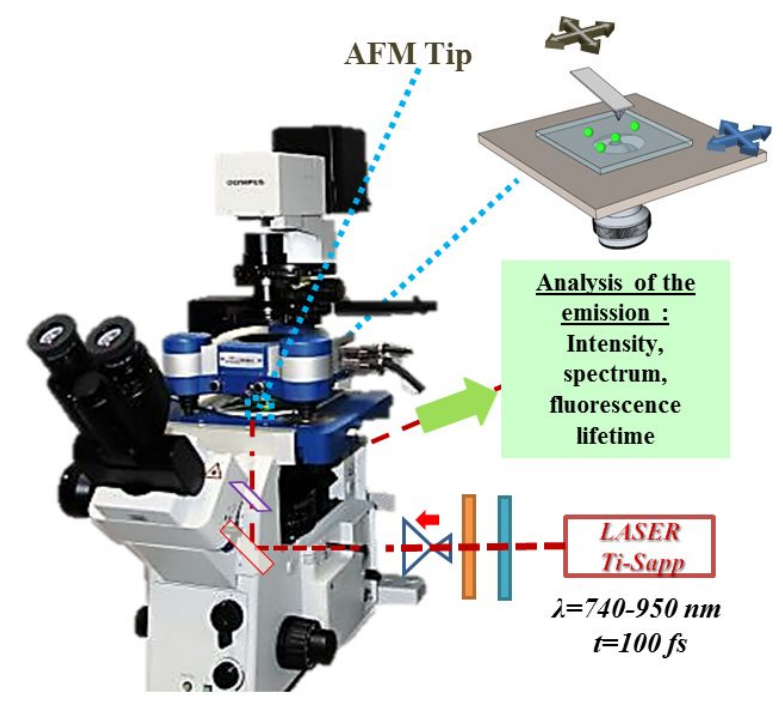

Figure 2: Coupling of a femtosecond laser with an AFM head (JPK NanoWizard III) through an inverted microscope (Olympus IX71). The laser (Tsunami Spectra Physics Inc.) delivers 100 fs pulses tunable from $740 \mathrm{~nm}$ to $950 \mathrm{~nm}$, The incoming laser beam is expanded in order to fill the aperture of the microscope objective (100x, $1.3 \mathrm{NA})$, which is used both for excitation of the NW and collection of their 2PL. As schematically represented in magnification between the dotted lines, this experimental setup involves two probes: the first probe is the AFM tip, for topography characterization (resolution limited by the tip apex size of a few tens of $\mathrm{nm}$ ) and the second is the focused laser beam, for 2PL studies (resolution directly linked to the beam waist at the focus measured about $0,5 \mu \mathrm{m}$ ): a key step in order to get correlated topography and 2PL images is the preliminary alignment of the AFM tip at the center of the focused laser spot.

Two-photon induced luminescence measurements were performed using an inverted microscope set-up coupled to a mode locked Ti-sapphire laser (Tsunami, Spectra Physics) delivering $100 \mathrm{fs}$ pulses with a $80 \mathrm{MHz}$ repetition rate over the spectral range covering 770 to $950 \mathrm{~nm}$. The laser beam is linearly polarized. Laser polarization together with average power can be controlled using sets of half-wave plate and polarizers. Special attention was taken concerning the choice of the power intensity at the level of the sample since gold nanowires are very sensitive and can easily be melted or burned once subjected to high power intensity ${ }^{11}$. The Ti-Saph excitation beam is focused onto samples using a $100 \mathrm{x}$ microscope objective (1.3 NA), the same objective being also used for the two-photon luminescence signal collection. The signal is then sent either to a channel plate multiplier working in the photon counting mode (Perkin Elmer MP-993CL) or to a spectrometer coupled to a CCD camera (Andor DU401-BR-DD) for detailed study of the emission spectra. Excitation spectra were determined from measurements of the whole emitted light using the previously mentioned CPM photomultiplier associated to a set of filters cutting the fundamental beam (SemRock razor edge 785, stopline 785 and 808, FF735 and FF-01-750).

As illustrated in figure 2, a cantilever type AFM platform is also combined to our 2PL microscopy set-up, enabling to perform correlated optical and topographic measurements. The tip can be raster scanned above the sample and vice versa, the sample can be raster scanned under a fixed tip. To correlate topographic and optical measurements, the requirement for this experiment is the preliminary alignment of the tip with the laser. 


\section{2 -2 Photoemission electron microscopy (PEEM) and low energy emission microscopy (LEEM) measurements}

Photoemission can be strongly enhanced upon excitation of surface plasmons (SPs). By collecting the photoemitted electrons, two-dimensional intensity maps reflecting the actual distribution of the optical near-field are obtained ${ }^{12}$. Important to note, this imaging technique makes use of well-established electron optics, i.e. involves no physical probe altering the measure. A routine spatial resolution of the order of $20 \mathrm{~nm}$ can be obtained.

The PEEM used (Elmitec GmbH) operates in ultra-high vacuum (in the order of $10^{-10} \mathrm{mbar}$ ) and uses extraction voltage in the order of the $\mathrm{kV}$. Optical excitation is delivered with grazing incidence $\left(75^{\circ}\right)$ by a laser beam from a cavity (Tsunami, Spectra Physics Inc.) tunable in the range $740 \mathrm{~nm}$ to $980 \mathrm{~nm}$. The duration of the pulse is $80 \mathrm{fs}$ and the repetition rate is $80 \mathrm{MHz}$. Unlike the experimental setup presented above, the laser beam is not focused, offering a wider field of view. The laser excites the plasmonic nanostructures resulting in oscillating electrons that are extracted and sent to a fluorescent screen. In parallel to PEEM measurements that provide plasmonic properties of the sample, LEEM images can also be acquired to get information about sample topography by sending accelerated electrons from a $\mathrm{LaB}_{6}$ tip to the sample. Figure 3 give a simplified representation of the PEEM / LEEM set-up.

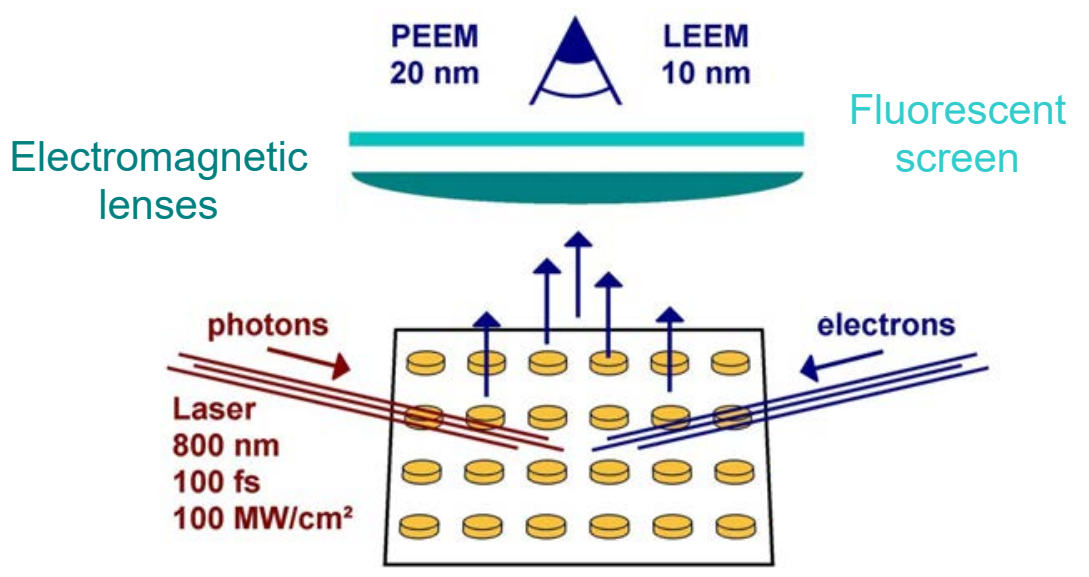

Figure 3: Principle of PEEM/LEEM measurements. Any plasmonic sample can be analysed like nanodisks in this case

\section{RESULTS AND DISCUSSION}

\section{3-1 2PL investigation of nanowires coming from colloidal chemistry}

Figure 4 shows the correlated topography and 2PL map obtained in the case of a given colloidal NW $(\mathrm{L}=930 \mathrm{~nm}, \mathrm{~W}=$ $220 \mathrm{~nm}, \mathrm{H}=140 \mathrm{~nm}$ ): these images were acquired simultaneously when raster scanning the nanowire immobilized onto an ITO covered thin slide after the preliminary careful alignment of the AFM tip with the focused laser spot. The exciting beam polarization was set parallel to the long axis of the NW, the average incident power being about $1 \mathrm{~mW}$.

Two bright luminescence spots can clearly be observed at both NW extremities revealing thus two hot spots and confirming previous observations from literature ${ }^{13}$.

The 2PL intensity is observed to be highly dependent on the polarization but also on the wavelength of the fundamental excitation beam which seems to indicate a quite low contribution of lightning-rod effects. As discussed previously, colloidal chemistry gives rise to quite a large variety of NW that happen to be either singly or multiply twinned ${ }^{14,15}$ and are observed to present more or less sharp extremities (see figure 1). Whatever the nanowire considered, the dependence of the hot spots 2PL intensity is always measured to be highly dependent on the exciting wavelength, consequently signing a plasmonic origin of the 2PL hot spots. 

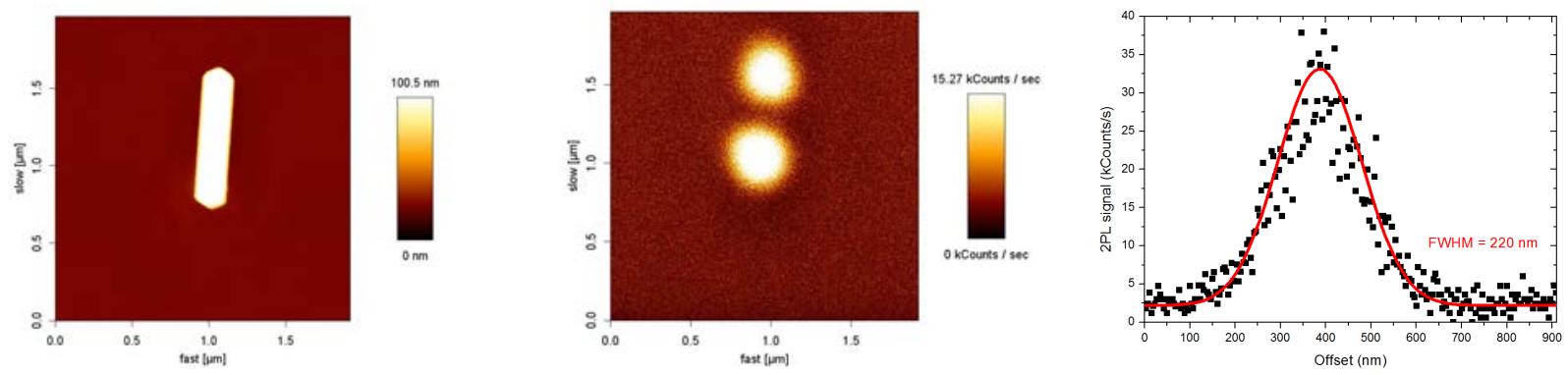

Figure 4: (left) Characterization of a colloidal NW (L = $930 \mathrm{~nm}, \mathrm{~W}=220 \mathrm{~nm}, \mathrm{H}=140 \mathrm{~nm})$ : correlated topography (tapping mode AFM) and (center) 2PL map obtained from raster scanning the NW. The NW is excited at $790 \mathrm{~nm}$ with an incident excitation power of $1 \mathrm{~mW}$. The incident polarization is oriented along the axis of the NW. When turning the incident polarization to a polarization perpendicular to the NW axis, the hotspots turn off: the 2PL intensity is uniform along NW with an overall intensity about $1 / 5$ of the $2 \mathrm{PL}$ intensity observed at the hot spots. Pixel integration time $=3.35 \mathrm{~ms}$. (right) Image showing the cross section of one the 2PL hot spots observed at the NW extremities: FWHM $=220 \mathrm{~nm}$, in fairly good agreement with the excitation beam waist at the sample $\mathrm{w}_{\omega}=0.4 \mu \mathrm{m}$ (quadratic dependence of 2PL ).

In the case of the wire studied in figure 4, the maximum 2PL hot spots is obtained for $\lambda_{\text {exc }}=790 \mathrm{~nm}$, which is in good agreement with the excitation of a third order Fabry-Perot mode considering equation (1). Although the resolution in 2PL microscopy prevents the direct observation of high order modes, this $3^{\text {rd }}$ order mode could interestingly be revealed taking into profit the high resolution capability of PEEM microscopy (see figure 5).
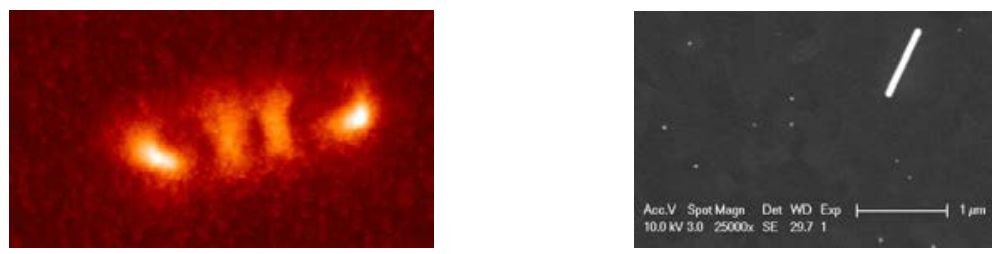

Figure 5: (Left) PEEM image obtained from a NW synthetized by CC. (Right) SEM image of the NW (915 nm x $95 \mathrm{~nm}$ )

\section{3-2 Comparative study of NW fabricated using e-beam lithography}

After characterizing colloidal NW of slightly similar length and aspect ratio, the properties of nanowires fabricated using e-beam lithography are investigated. In contrast to the case of CC nanowires, tapping mode AFM characterization of such wires reveals quite high roughness (see left part of figure 6).

Correlated 2PL mapping of these wires reveal however quite similar features as in the case of CC NW. In a the case of an exciting laser beam with a polarization along the NW axis, 2 bright 2PL spots are observed at both NW extremities, these spots vanishing for an exciting beam polarization perpendicular to the wire axis (uniform 2PL along the NW).
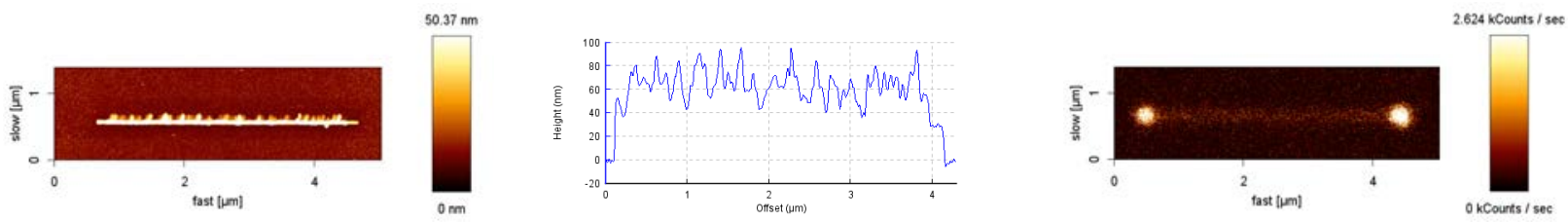

Figure 6: (left) Example of the topography of a NW made by EBL using the AFM in tapping mode. The dimensions of the NW are: $\mathrm{L}=4 \mu \mathrm{m}, \mathrm{W}=35 \mathrm{~nm}$. (center) The average roughness is about $10 \mathrm{~nm}$ and an average height $\mathrm{H}$ of $70 \mathrm{~nm}$ was determined by taking a cross-section along the NW. (right) 2PL cartography of the same area, the hot spots are again localized at the extremities of the NW. The image is acquired with an incident power of $250 \mu \mathrm{W}$ before entering the microscope at $800 \mathrm{~nm}$ and with an incident polarization along the axis of the NW. 
As mentioned above, the electric-field enhancement due to the plasmon mode resonance is the origin of the TPL increase at the hot spots. According to the theoretical formulation of photoluminescence from the surfaces of metallic films, 2PL intensity can approximately be given by Boyd et $\mathrm{al}^{16}$. and Chen et al. ${ }^{17}$.

$$
I_{T P L} \propto L^{4}(\omega) L^{2}\left(\omega_{2 P L}\right),
$$

where $\mathrm{L}(\omega)$ and $\mathrm{L}\left(\omega_{2 \mathrm{PL}}\right)$ denote local field correction factors for incident (fundamental frequency $\omega$ ) and emitted photons (2PL frequency $\omega_{2 \mathrm{PL}}$ ), respectively.

As a consequence, considering the ratio between the 2PL intensity measured at resonance (hot spot at the NW extremity for a polarization parallel to the wire: $I_{2 P L}^{\prime \prime}$ ) or out of resonance (2PL obtained for a polarization perpendicular to the $\mathrm{NW}: \perp_{2 P L}$ ) enables thus a direct evaluation of the field enhancement in intensity at the wires ends: $\mathrm{FE}^{2}=I_{2 P L} / I_{2 P L}$. After analysis of a few tens of objects, both types of wires, either coming from colloidal chemistry or EBL are observed to present comparable field enhancement effects, meaning that crystallinity or inversely, roughness seem to be of no influence. Finally, unlike most predictions in literature, FE happens also to be quite limited: in average FE is found to be around $3:<\mathrm{FE}>\sim 3<<100-1000^{18}$.

Additional experiments are currently under way towards the investigation of a larger number of NW in order to optimize the statistics of the results.

\section{3-3 Emission spectra coming from the hot spots}

As a complementary characterization, the light emission at the hot spots was analyzed spectrally for both types of NW (figure 7). Two contributions can clearly be distinguished: a quite sharp peak at $2 \omega$ (not visible for the case of the NW made by EBL), which can be attributed to gold surface second harmonic generation and a broad luminescence band.

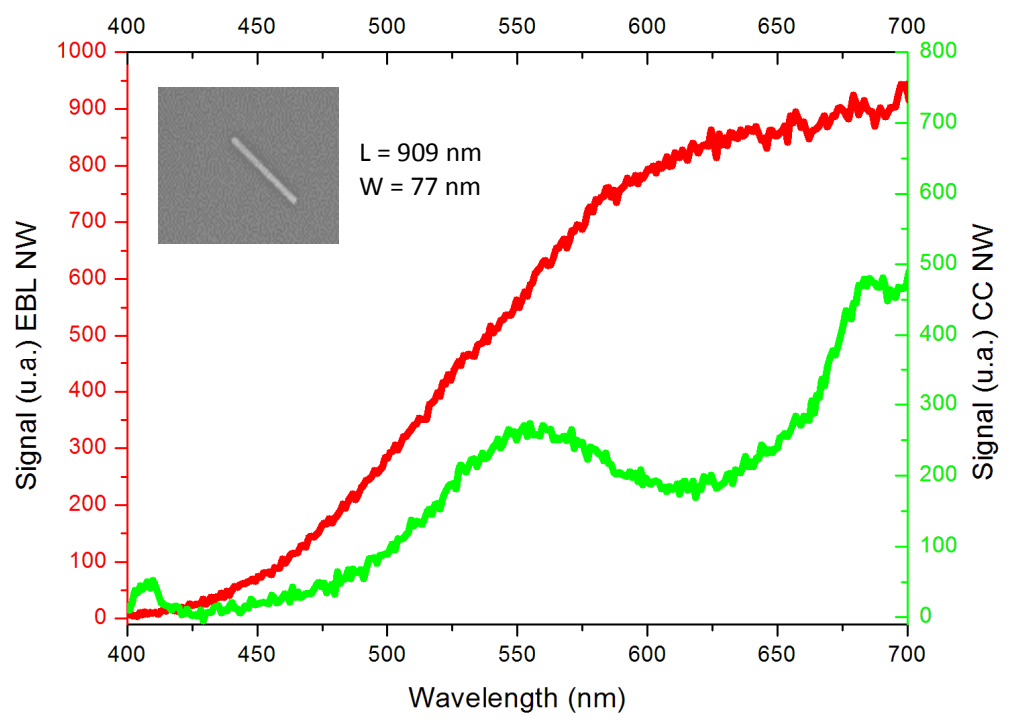

Figure 7: Emission spectrum coming from a hot spot at the surface of a: (right scale, green line) CC NW (the inset shows a SEM image of the considered NW) and (left scale, red line) EBL NW (SEM characterization shown in figure 1). No information is available for wavelength greater than $700 \mathrm{~nm}$, the optical signal being filtered in order to cut off any residual contribution from the incident laser excitation.

As evidenced in figure 7, the luminescence spectrum of both types of NW happen to be strikingly different: whereas EBL NW give rise to a quite broad and unstructured emission spectrum mostly centered in the red to IR, colloidal NW all exhibits structured emission spectra with multiple and well-defined peaks. In the case of the colloidal NW reported in 
figure 7, two clear peaks can be noticed, one around $560 \mathrm{~nm}$ and one around $680 \mathrm{~nm}$. These two peaks are well known to correspond to the energy gap between the d-band and the Fermi level of gold at the so-called L and X symmetry point of the first Brillouin zone (Imura et al. ${ }^{19}$ ). Together with revealing the crystalline structure of the NW, such results confirm that 2PL results from a radiative recombination of electron-hole pairs generated following absorption processes consecutive to the NW excitation at resonance.

Oppositely, in the case of EBL NW no peaks can be observed since the sample has a certain roughness and no specific crystalline face is privileged.

This seems to be in contradiction with previously reported experiments in the team of D. Brida ${ }^{20}$. In the experiments reported by Brida and coworkers, the objects considered were however quite different, together with the excitation conditions, making direct comparison of the results quite complex.

\section{CONCLUSION}

Complementary AFM/2PL measurements were performed to investigate field enhancement effects at different type of nanowires either made using colloidal chemistry or electron-beam lithography. 2PL hot spots are localized at the extremities of the NW, the ends of the rods acting as point like detection probes. The 2PL intensity at the hot spots is observed to be highly dependent on the polarization but also on the wavelength of the fundamental excitation beam: we conclude that the main contribution to the hot spots are plasmonic effects, the contribution of lightning-rod effects being mostly negligible. Although 2PL lacks resolution to observe the higher modes of resonance, PEEM characterization was performed revealing the excitation of higher order mode of resonance (order 3). Several NW have been studied and rather limited field enhancement effects were found, both for CC or EBL nanowires : $\left(\mathrm{FE}_{\mathrm{CC}}\right) \sim\left(\mathrm{FE}_{\mathrm{EBL}}\right) \sim 3$ to 4 , which is surprisingly much lower than values deduced from numerical simulations of idealized NW. Quite interestingly, the effect of the wire crystallinity, was evidenced: whereas EBL NW give rise to a quite broad and unstructured emission spectrum, colloidal NW exhibit structured emission spectra with multiple and well-defined peaks that can be assigned to interband transition in gold, further confirming that 2PL results from a radiative recombination of electron-hole pairs generated following absorption processes consecutive to the NW excitation at resonance.

\section{Acknowledgments}

The authors wish to thank the Agence Nationale de la Recherche (ANR) and C'Nano IdF program for their financial support to this work through the project ANR-13-nano-SAMIRé and INPACT, respectively. J. Béal is also gratefully acknowledged for the realization of grids for the specific identification of individual colloidal nanowires from SEM to AFM characterization.

\section{REFERENCES}

[1] Atwater, H. A. and Polman, A., "Plasmonics for improved photovoltaic devices." Nat. Mater. 9, 205-213 (2010).

[2] Stewart, M. E., Anderton, C. R., Thompson, L. B., Maria, J, Gray, S. K, Rogers, J. A. and Nuzzo, R. G., "Nanostructured Plasmonic Sensors." Chem. Rev. 108, 494-521 (2008).

[3] Hill, M. T. and Gather, M. C., "Advances in small lasers." Nat. Photonics 8, 908-918 (2014).

[4] Kelly, K. L., Coronado, E., Zhao, L. L. and Schatz, G. C., "The Optical Properties of Metal Nanoparticles: The Influence of Size, Shape, and Dielectric Environment." J. Phys. Chem. B 107, 668-677 (2003).

[5] Novotny, L., Bian, R. X. and Xie, X. S., "Theory of nanometric optical tweezers." Phys. Rev. Lett. 79, 645-648 (1997).

[6] Verellen, N., Denkova, D., Clercq, B.D., Silhanek, A.V., Ameloot, M., Dorpe, P.V. and Moshchalkov, V., "TwoPhoton Luminescence of Gold Nanorods Mediated by Higher Order Plasmon Modes." ACS Photonics 2, 410-416 (2015).

[7] Imura, K. and Okamoto, H., "Properties of Photoluminescence from Single Gold Nanorods Induced by Near-Field Two-Photon Excitation". J. Phys. Chem. C 113, 11756-11759 (2009).

[8] Sau, T. K. and Murphy, C. J., "Seeded High Yield Synthesis of Short Au Nanorods in Aqueous Solution." Langmuir 20, 6414-6420 (2004). 
[9] Sönnichsen, C., Franzl, T., Wilk, T., Von Plessen, G., Feldmann, J., Wilson, O. and Mulvaney, P., "Drastic Reduction of Plasmon Damping in Gold Nanorods." Phys. Rev. Lett. 88, 077402 (2002).

[10] Viarbitskaya, S, Teulle, A., Marty, R., Sharma, J., Girard, C., Arbouet, A. and Dujardin, E., "Tailoring and imaging the plasmonic local density of states in crystalline nanoprisms." Nat. Mater. 12, 426-432 (2013).

[11]Zijlstra, P., Chon, J. W. M. and Gu, M., "White light scattering spectroscopy and electron microscopy of laser induced melting in single gold nanorods." Phys. Chem. Chem. Phys. 11, 5915-5921 (2009).

[12] Douillard, L. and Charra, F., "High-resolution mapping of plasmonic modes: photoemission and scanning tunnelling luminescence microscopies." J. Phys. Appl. Phys. 44, 464002 (2011).

[13] Wissert, M. D., Ilin, K. S., Siegel, M., Lemmer, U. and Eisler, H.-J., "Highly localized non-linear optical white-light response at nanorod ends from non-resonant excitation." Nanoscale 2, 1018-1020 (2010).

[14] Pérez-Juste, J., Pastoriza-Santos, I., Liz-Marzán, L. M. and Mulvaney, P., "Gold nanorods: Synthesis, characterization and applications." Coord. Chem. Rev. 249, 1870-1901 (2005).

[15] Sau, T. K., Rogach, A. L., Jäckel, F., Klar, T. A. and Feldmann, J., "Properties and Applications of Colloidal Nonspherical Noble Metal Nanoparticles." Adv. Mater. 22, 1805-1825 (2010).

[16] Boyd, G. T., Yu, Z. H. and Shen, Y. R., "Photoinduced luminescence from the noble metals and its enhancement on roughened surfaces." Phys. Rev. B 33, 7923-7936 (1986).

[17] Chen, C. K., de Castro, A. R. B. and Shen, Y. R., "Surface-Enhanced Second-Harmonic Generation." Phys. Rev. Lett. 46, 145-148 (1981).

[18]Zijlstra, P., Paulo, P. M. R. and Orrit, M., "Optical detection of single non-absorbing molecules using the surface plasmon resonance of a gold nanorod." Nat. Nanotechnol. 7, 379-382 (2012).

[19] Imura, K., Nagahara, T. and Okamoto, H., "Near-Field Two-Photon-Induced Photoluminescence from Single Gold Nanorods and Imaging of Plasmon Modes." J. Phys. Chem. B 109, 13214-13220 (2005).

[20] Knittel, V., Fischer, M. P., de Roo, T., Mecking, S., Leitenstorfer, A. and Brida, D, "Nonlinear Photoluminescence Spectrum of Single Gold Nanostructures." ACS Nano 9, 894-900 (2015). 